\title{
Correction to: Development and Research of a Terminal Controller for Marine Robots
}

V. Pshikhopov and Boris Gurenko

\section{Correction to:}

Chapter "Development and Research of a Terminal Controller for Marine Robots" in: H. Fujita et al. (Eds.):

Trends in Artificial Intelligence Theory and Applications, LNAI 12144, https://doi.org/10.1007/978-3-030-55789-8_76

The original version of this chapter was revised. The number in the funding statement was corrected to MK- 3099.2019.8. 